\title{
Politique d'infrastructure et choix de localisation dans un modèle de signal
}

\author{
Jean-Philippe Tropeano* \\ EUREQua, UMR CNRS-Université de Paris $I^{* *}$
}

\section{Introduction}

"La mode, c'est Paris». C'est par cette formule lapidaire que M. Jean Bousquet annonce et explique le transfert du siège de Cacharel de Nìmes à Paris ${ }^{1}$. Pinçon et Pinçon-Charlot (1992) insistent sur l'extrême concentration spatiale des entreprises : ça n'est pas Paris qui est le lieu privilégié de la localisation des industries du luxe mais le VIII ème arrondissement. Ils montrent la volonté délibérée des entreprises de s'implanter au plus près de leurs concurrents. M. Alain Boucheron, joaillier Place Vendôme, affirme : « il y a une image mondiale de la place Vendôme et de la rue de la Paix pour la haute joaillerie. Venir s'y installer, pour un joaillier, c'est rejoindre la cour des grands. » Les deux sociologues ajoutent : « la griffe spatiale impose sa loi et l'on se doit d'être spatialement proche des concurrents dont la proximité, paradoxalement, assoit votre propre notoriété ». Dans un domaine radicalement différent, l'entreprise Mil Cristal, leader des prothèses en céramique, justifie sa localisation à Limoges en soulignant le « label » associé à la ville dans le secteur de la céramique ${ }^{2}$.

L'agglomération spatiale des firmes est un phénomène bien connu de l'économie géographique. Les « districts industriels » regroupant des entreprises de céramique, de jouets, d'électronique, d'aéronautique...sont nom-

\footnotetext{
* L'auteur remercie tout particulièrement Anne Perrot ainsi que deux rapporteurs anonymes.

** 106-112, boulevard de l'Hópital, 75647 Paris Cedex 13, France.

tropeano@univ-paris1.fr

1 Le Monde du 2 décembre 1997.

2 Les Echos, lundi 19 avril 1999.
} 
breux en Italie du nord, en France, aux États-Unis...(OCDE (1996), Porter (1998)). A Tokyo, par exemple, la plupart des firmes sous-traitantes produisant des composants électroniques haut de gamme pour les grands groupes japonais se concentrent dans un quartier périphérique. Malgré la forte intégration des marchés financiers et le développement des technologies de l'information qui diminuent le coût et augmentent la vitesse de transmission de l'information, la concentration spatiale des firmes bancaires se renforce. Gehrig (1998) montre plus précisément que si les activités traditionnelles ont tendance à être délocalisées en fonction d'avantages fiscaux ou salariaux, les nouveaux produits financiers sont toujours d'abord développés dans quelques grands centres. En effet, lorsqu'un nouveau produit financier est mis en vente, les informations sur le risque ou le rendement réel de ce produit restent informelles. Les agents ne peuvent se procurer ce type de renseignements que par une relation directe. La distance introduit un bruit dans la transmission de l'information sur le produit et altère sa qualité.

Des études empiriques plus systématiques confirment la concentration spatiale des firmes qu'elles soient issues d'un même secteur d'activité ou non (Glaeser (1997)).

Les raisons invoquées traditionnellement pour expliquer cette forte concentration spatiale sont au nombre de trois (Marshall (1920)). D'abord, la proximité spatiale permet aux firmes de profiter d'externalités technologiques. Ensuite, la polarisation géographique permet aux firmes de minimiser les coûts de transport en se localisant à proximité des consommateurs ou des fournisseurs. Enfin, une firme augmente les chances d'embaucher une main d'oeuvre adaptée en se localisant dans une région qui regroupe déjà un grand nombre de firmes.

Par ailleurs, plusieurs études empiriques mettent aussi en évidence la concurrence comme facteur d'innovation (Blundell, Griffith et Van Reenen (1995)) et de localisation de firmes innovantes (Audretsch et Feldman (1999)). Ainsi, plus la concurrence est intense, plus les firmes sont incitées à innover mais surtout plus la concurrence est vive au sein d'une région, plus de nouvelles entreprises ont tendance à s'y implanter.

Les modèles théoriques (Aghion et Howitt (1997)) expliquent le premier constat en montrant que la concurrence contraint les entreprises à innover sous peine de périr. Shulz et Stahl (1996) invoquent un autre argument pour montrer que la concurrence peut être un facteur d'agglomération. Ils soulignent que lorsque les consommateurs acquittent des coûts de prospection, ces derniers se dirigent vers la région où se concentrent le plus grand nombre de firmes. Afin de profiter d'une demande importante, les entreprises peuvent être ainsi incitées à se localiser dans la région où la concurrence est intense.

Toutefois, dans les exemples précédents, les entreprises semblent mettre aussi en avant la localisation comme le moyen de signaler des caractéristiques (qualité, variété...) de biens nouveaux encore peu connus des consommateurs. Les entrepreneurs semblent dire : « accepter la concurrence en 
se localisant au plus près des entreprises existantes permet de prouver aux consommateurs la valeur des produits vendus ». La polarisation géographique et par là même une plus grande concurrence sert de faire-valoir aux innovations. Une ville ou une région où les consommateurs disposent déjà d'une grande variété de biens est. un lieu propice au développement de produits nouveaux. La diversité source de croissance et de développement est mise en évidence par Jacobs (1969 et 1984) ou encore récemment par Quigley (1998). La raison invoquée dans cet article est la suivante : il est plus facile de signaler le caractère novateur d'un bien dans un environnement où les consommateurs ont déjà accès à une gamme de biens importante. Si une nouvelle firme choisit, malgré cette forte concurrence, de développer le bien dans un tel environnement, cela assure la valeur de l'innovation aux yeux des consommateurs. Par exemple, les fondateurs de la firme Nexpert, spécialisée dans les logiciels d'aide au diagnostic médical, n'ont pas réussi à développer leur produit à partir du marché français. Seule une firme californienne a accepté de financer le projet. Ce n'est que par la suite que le produit a été vendu en France ${ }^{3}$. Les dirigeants de la firme affirment que les réticences des banques françaises s'expliquaient par un manque d'information sur l'utilité que pouvait avoir pour les médecins un logiciel d'aide au diagnostic. La réussite en Californie assurait en revanche l'intérêt que pouvait revêtir ce bien auprès des consommateurs.

Dans un tel modèle où la localisation sert de signal pour une innovation et où par conséquent les entreprises se polarisent géographiquement, on étudie l'impact d'une baisse des coûts de transport sur la localisation des firmes innovantes ou existantes. On se demande en particulier si une baisse des coûts de transport renforce ou au contraire atténue l'effet de signal de la localisation.

Les principales conclusions de cet article sont les suivantes.

On montre que l'agglomération est un instrument de signal pour les entreprises. Plus précisément, en l'absence d'asymétrie d'information, une firme entrante n'a aucune incitation à se localiser dans la même région que la firme installée alors que la présence d'asymétrie d'information incite la firme entrante à s'implanter à proximité de la firme en place pour signaler la qualité de son produit. En d'autres termes, une incertitude des consommateurs sur la qualité des nouveaux produits est l'unique source de polarisation des entreprises au sein d'une même région ${ }^{4}$.

Par ailleurs, nous soulignons que cette forte polarisation doit inciter les autorités supra-régionales à éviter toute politique de «saupoudrage " des investissements entre différentes régions. En effet, dans ce modèle, un investissement modeste se traduisant par une faible diminution du coût

3 Le Monde, mercredi 28 avril 1999.

4 Bester (1998) montre également que l'asymétrie d'information se traduit par une polarisation spatiale des firmes. Toutefois son analyse est radicalement différente. Les firmes signalent la qualité des biens par des prix élevés. Ce reláchement de la concurrence permet alors une agglomération spatiale sur le segment. En revanche le présent article se situe dans la même lignée que Bester (1998) dans la mesure où il se concentre sur le rôle de la localisation comme signal destiné aux consommateurs et non aux investisseurs. 
de transport renforce la polarisation des firmes en augmentant, en conséquence les coûts de transport totaux. Le résultat peut être une diminution du bien-être total. Les autorités publiques doivent donc privilégier des infrastructures de désenclavement qui permettent de rapprocher franchement les villes plutôt que de multiplier les investissements.

La section 2 présente le modèle. On étudie la localisation des firmes d'abord en information parfaite (section 3) puis en information incomplète (section 4) avant d'examiner dans la section 5 les implications en termes de bien-être d'une baisse des coûts de transport.

\section{$2 \quad$ Le Modèle}

On considère deux régions, une région Nord $N$ et une région Sud $S$, reliées entre elles par une infrastructure de transport. On appelle $\lambda$ le coût unitaire de transport nécessaire pour emprunter cette infrastructure inter-régionale.

On normalise à 1 le nombre d'agents installés dans chacune des deux régions.

L'utilité indirecte d'un agent est la suivante:

$$
U(q, P)= \begin{cases}q-P & \text { si } q-P \geqslant 0 \\ 0 & \text { sinon }\end{cases}
$$

où $P$ désigne le prix du bien vendu par une entreprise produisant un bien de qualité $q$.

Les firmes adoptent une politique de prix rendu et supportent donc les coûts de transport. Une entreprise s'implante dans une seule région. Les coûts de relocalisation sont supposés infinis : le choix de localisation est définitif. Les firmes ne supportent pas de coût de production. Aucun élément ne distingue donc la région $N$ de la région $S$.

Le bien considéré est un bien d'expérience : les consommateurs ne connaissent pas a priori sa qualité. Ils ne la découvrent qu'une fois le bien consommé. L'achat de ce bien est répété au cours du temps. Cet élément est capital. En effet, lorsque les consommateurs prennent connaissance de la réelle qualité du bien, la firme ne peut modifier sa localisation. On suppose le paramètre d'actualisation nul. L'objectif des firmes est donc de maximiser la somme des profits.

Une firme $I$ est déjà présente sur le marché et est supposée localisée dans la région Nord. Elle vend un bien de qualité $q^{I}$ connue de tous. En revanche, les consommateurs comme la firme installée ne connaissent pas la qualité, $q$, du bien vendu par le nouvel entrant $E$. Cette entreprise a engagé des dépenses de $R \& D$ dont le but est d'augmenter la qualité du bien. Le résultat de la recherche est aléatoire et ex-ante l'issue de cette dépense de $\mathrm{R} \& \mathrm{D}$ est représentée par la fonction de densité $f(q)$. Le montant de ces 
dépenses est observable par les consommateurs et la firme installée. Ces derniers agents ignorent la qualité ex-post mais connaissent la technologie de $R \& D, f(q)$, qui représente donc les croyances a priori des agents sur le résultat de l'investissement en $\mathrm{R} \& \mathrm{D}$. Nous supposons que les dépenses en $\mathrm{R} \& \mathrm{D}$ permettent à la firme entrante d'avoir accès à la technologie utilisée par la firme installée. La qualité produite est donc supérieure ou égale à $q^{I}$ $\left(q \in\left[q^{I}, \bar{q}\right]\right) . f(q)$ vérifie $^{5}$ donc:

$$
\int_{q^{I}}^{\bar{q}} f(q) d q=1
$$

On note $q^{m}$ la qualité moyenne ex-ante de la firme innovante:

$$
q^{m}=\int_{q^{1}}^{\bar{q}} q f(q) d q
$$

On étudie dans un premier temps la localisation de l'entreprise entrante en information complète avant de la comparer à la localisation en information incomplète.

\section{Localisation en information complète}

Prenons comme référence le jeu en information complète. Le déroulement du jeu est précisé sur le graphique suivant (figure 1).

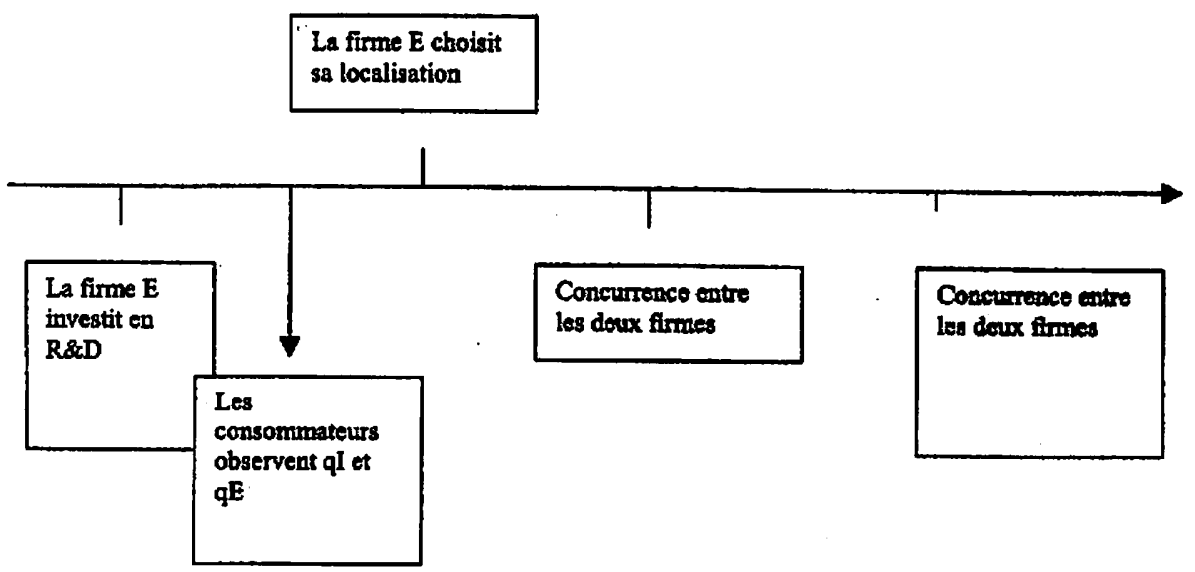

Figure 1 : Jeu en information complète

5 On suppose $\bar{q}>4 q^{l}$. 
Au cours d'une étape antérieure, une première firme, $I$, s'est implantée dans la région Nord.

En première étape, une firme $E$ entre sur le marché en proposant un produit de qualité $q$ connue des consommateurs. La firme $E$ détermine sa localisation $e$.

Dans une deuxième étape, les deux firmes se font concurrence en prix à la Bertrand. À la troisième étape, cette concurrence en prix est répétée.

Le jeu se résout par induction vers l'amont. Déterminons par conséquent l'équilibre en prix des périodes deux et trois. Remarquons que le nombre de périodes étant fini, le seul équilibre du jeu répété en prix est la simple répétition de l'équilibre du jeu en prix à une période.

Deux cas sont à étudier en fonction de la localisation de la firme entrante.

(i) L'entreprise $E$ est localisée en $N$.

Les firmes adoptant une politique de prix rendu, la détermination d'un prix sur un marché ne dépend pas du prix fixé sur l'autre marché. On note $P_{i}^{E}$ le prix de la firme entrante et $P_{i}^{\prime}$ le prix de la firme installée sur le marché $i(i=N, S)$. Si l'on note $t_{i}$ le coût de transport unitaire supporté par les firmes pour desservir le marché $i\left(t_{N}=0\right.$ et $\left.t_{S}=\lambda\right)$, les prix d'équilibre $\left(P_{i}^{E}\right)^{*}$ et $\left(P_{i}^{I}\right)^{*}$ sont par conséquent ${ }^{6}$ :

$$
\begin{aligned}
& \left(P_{i}^{E}\right)^{*}= \begin{cases}q & \text { si } t_{i}>q^{I} \\
q-q^{I}+t_{i} & \text { si } t_{i}<q^{I}\end{cases} \\
& \left(P_{i}^{I}\right)^{*}=t_{i}
\end{aligned}
$$

Ces prix d'équilibre s'interprètent aisément : si le coût de transport ne permet pas à la firme installée de servir le marché Sud $\left(\lambda>q^{I}\right)$, la firme entrante vend à son prix de monopole $(q)$ sur ce marché. Dans la cas contraire, la firme entrante vend au prix limite assurant l'indifférence des consommateurs entre les deux biens.

On note $\Pi^{E}\left(e, P^{E}, q\right)$ le profit total (marchés Nord et Sud) de la firme entrante lorsqu'elle vend un bien de qualité $q$ et est localisée en $e$. Le profit d'équilibre $\Pi^{E}\left(N,\left(P^{E}\right)^{*}, q\right)$ est par conséquent :

$$
\Pi^{E}\left(N,\left(P^{E}\right)^{*}, q\right)= \begin{cases}q-q^{I} & \text { si } \lambda>q \\ \left(q-q^{I}\right)+(q-\lambda) & \text { si } q^{I}<\lambda<q \\ \left(q-q^{I}\right)+\left(q-q^{I}\right) & \text { si } \lambda<q^{I}\end{cases}
$$

Remarquons qu'un coût de transport très élevé $(\lambda>q)$ empêche la firme entrante de desservir le marché Sud.

(ii) L'entreprise entrante est localisée en $S$.

6 Ce résultat est explicité dans l'annexe 1. II s'agit d'un cas très simple de concurrence à la Bertrand avec coût et qualité asymétriques. 
En adoptant les mêmes notations que précédemment, les prix d'équilibre sont par conséquent sur le marché Sud:

$$
\begin{aligned}
& \left(P_{S}^{E}\right)^{\times}= \begin{cases}q & \text { si } \lambda \geqslant q^{I} \\
\left(q-q^{\prime}\right)+\lambda & \text { si } \lambda \leqslant q^{I}\end{cases} \\
& \left(P_{S}^{I}\right)^{\times}=\lambda
\end{aligned}
$$

Un coût de transport prohibitif $\left(\lambda \geqslant q^{I}\right)$ permet à la firme entrante d'être en monopole sur le marché $S$. Dans le cas contraire, la firme entrante profite du coût de transport supporté par l'entreprise installée et majore la différence de qualité de ce coût.

Sur le marché Nord :

$$
\begin{aligned}
& \left(P_{N}^{E}\right)^{*}= \begin{cases}q-q^{l} & \text { si } q \geqslant q^{l}+\lambda \\
\lambda & \text { sinon }\end{cases} \\
& \left(P_{N}^{l}\right)^{*}= \begin{cases}\lambda & \text { si } q \geqslant q^{l}+\lambda \\
q^{l} & \text { sinon }\end{cases}
\end{aligned}
$$

Sur le marché Nord, un coût de transport excédant la différence de qualité entre la firme installée et la firme entrante empêche cette dernière d'entrer sur le marché.

Le profit total de la firme entrante est par conséquent : $-\operatorname{Si} q^{J}>\lambda$

$$
\Pi^{E}\left(S,\left(P^{E}\right)^{*}, q\right)= \begin{cases}q & \text { si } \lambda>q-q^{I} \\ q-q^{I}-\lambda+q & \text { si } \lambda<q-q^{I}\end{cases}
$$

Dans ce cas, la firme installée ne peut entrer sur le marché Sud sur lequel la firme entrante se trouve alors en monopole. Le niveau de coût de transport permet ou empêche la firme entrante de pénétrer sur le marché Nord.

- Si $q^{I}<\lambda$

$$
\Pi^{E}\left(S,\left(P^{E}\right)^{*}, q\right)= \begin{cases}q-q^{I}+\lambda & \text { si } \lambda>q-q^{I} \\ \left(q-q^{I}-\lambda\right)+\left(q-q^{I}+\lambda\right) & \text { si } \lambda<q-q^{I}\end{cases}
$$

Ici, la firme installée peut toujours concurrencer la firme entrante. Par ailleurs, un coût de transport élevé (ou un écart de qualité faible : $\lambda>q-q^{I}$ ) ne permet pas à la firme entrante d'entrer sur le marché Nord.

Remarquons toutefois que dans les deux cas, les profits ont un profil identique : si l'écart de qualité est suffisamment important la firme entrante réalise un profit sur les deux marchés.

À la deuxième étape du jeu, la firme entrante détermine sa localisation. Les figures 2 et 3 représentent le profit de la firme entrante selon la 


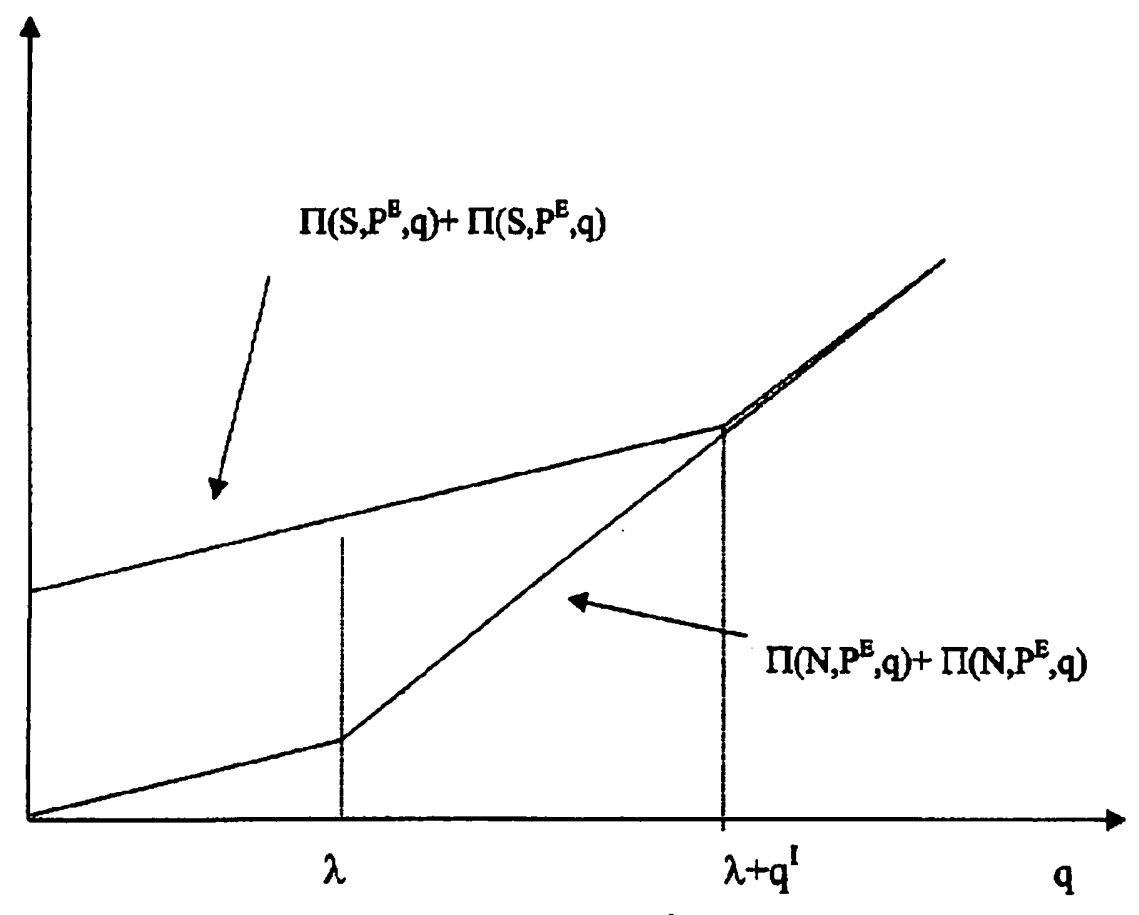

Figure 2: $q^{I}<\lambda$

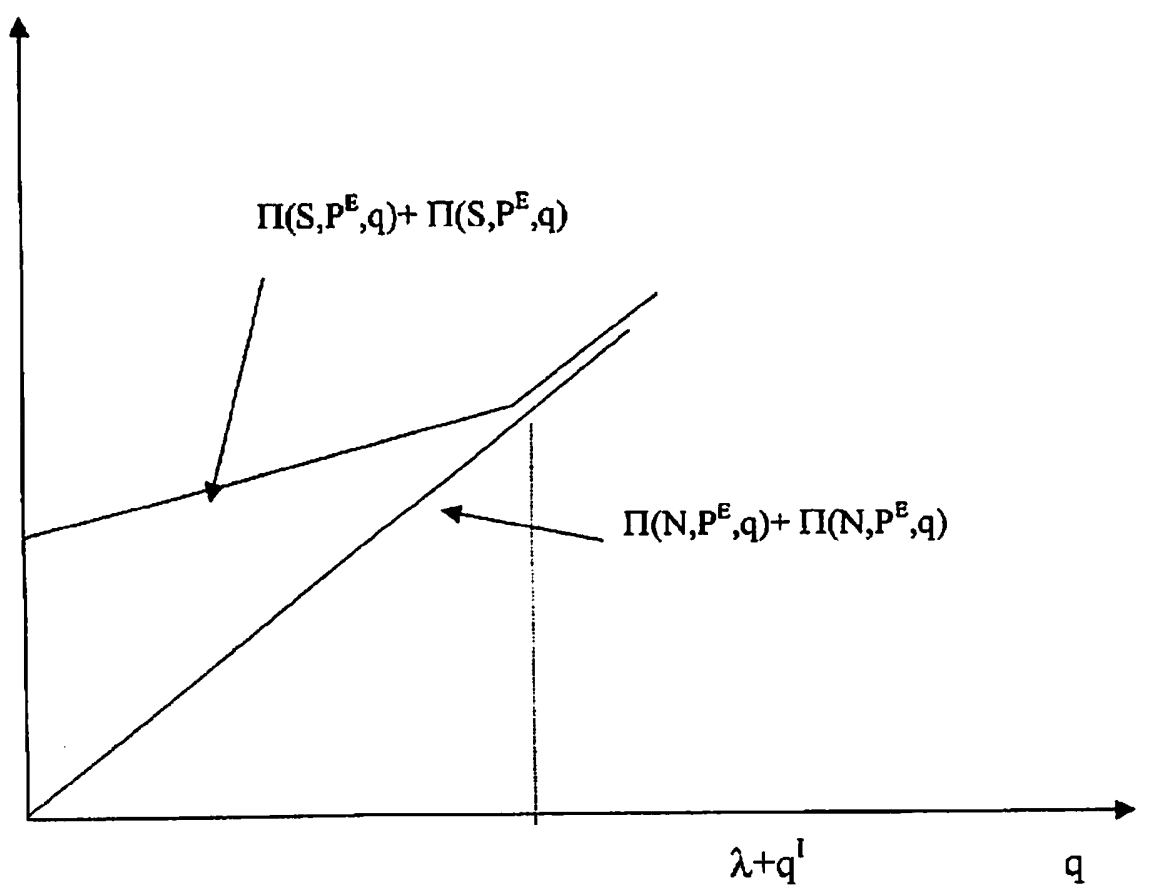

Figure $3: q^{l}>\lambda$ 
localisation choisie en fonction de la qualité $q$. On distingue le cas d'une firme installée pouvant concurrencer la firme entrante sur le marché Sud $\left(q^{I}>\lambda\right)$ du cas d'une firme installée ne pouvant desservir le marché Sud $\left(q^{I}<\lambda\right)$.

Dans les deux configurations, la concurrence exercée par la firme installée sur le marché Nord incite toujours la firme entrante à se localiser dans la région Sud. La raison est simple : le coût de transport supporté par la firme installée sur le marché Sud permet à la firme entrante de réaliser une marge plus élevée lorsqu'elle est elle même localisée sur ce marché : $q-q^{I}+\lambda$ au lieu de $q-q^{I}$. Seul un niveau très élevé de la qualité rend au mieux l'entreprise entrante indifférente. En d'autres termes, comme dans l'ensemble des modèles d'économie géographique (Ottaviano et al. (2002)), la dispersion spatiale permet de relâcher la concurrence.

Proposition 1 En information complète, si la qualité de la firme entrante est inférieure à une certaine borne, notée $\hat{q}(\lambda)$, cette firme s'implante dans la région Sud.

\section{Preuve}

Si $q^{I}>\lambda$, la comparaison des expressions (6) et (11) nous permet de conclure que la firme $E$ préfère s'implanter dans la région Sud dès que $q \leqslant q^{I}+\lambda=\hat{q}(\lambda)$.

Si $q^{I}<\lambda$, la comparaison des expressions (6) et (12) nous permet de conclure que la firme $E$ préfère s'implanter dans la région Sud dès que $q \leqslant \lambda+q^{I}=\hat{q}(\lambda)$.

Ce résultat n'est en rien surprenant et nous permet au contraire de mieux souligner le rôle de l'asymétrie d'information comme force de polarisation.

La section suivante résout le jeu en information incomplète.

\section{$4 \quad$ Localisation en information incomplète}

Le jeu se déroule comme précédemment. Toutefois, contrairement à la section précédente, la qualité de la firme entrante est inobservée par les consommateurs et par la firme installée. On considère toujours que la firme installée est localisée en $N$. sation $e$.

En première étape, le nouvel entrant cherche à déterminer sa locali-

Dans une deuxième étape, les deux firmes se font concurrence en prix à la Bertrand.

Au cours de la troisième étape, les consommateurs prennent connaissance de la réelle qualité du bien de la firme entrante. Ils répètent leur achat, les firmes se font à nouveau concurrence à la Bertrand. 


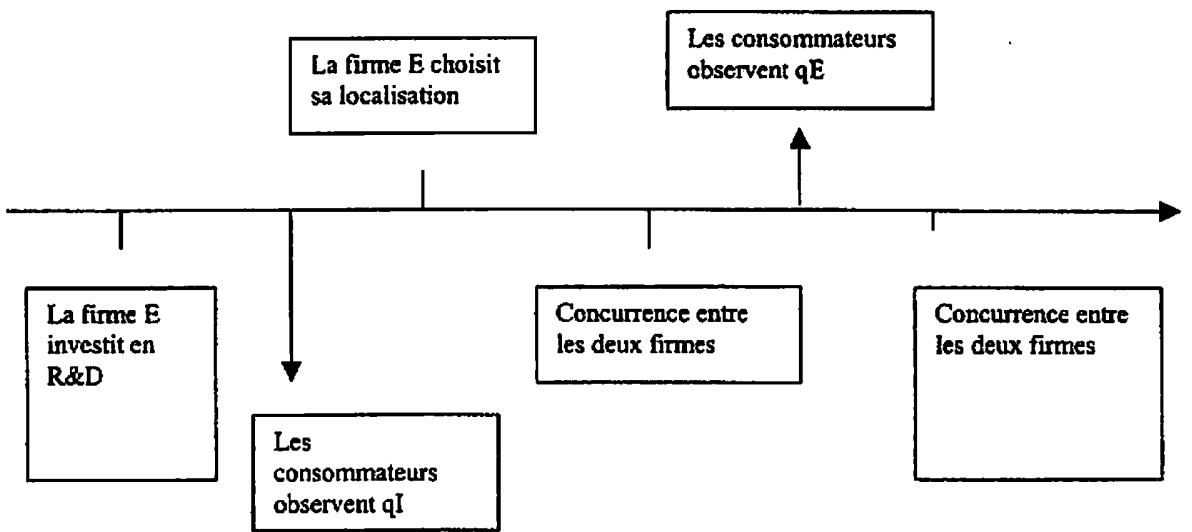

Figure 4 : Jeu en information incomplète

On peut résumer le déroulement du jeu par la figure 4 .

Les consommateurs révisent leur croyances à l'issue de l'étape 2 en fonction de la stratégie de la firme entrante. Soient $f\left(q \mid P_{2}^{E}, e\right)$ ces nouvelles croyances où $P_{2}^{E}$ désigne le prix pratiqué par la firme $E$ à l'étape $2^{7}$. On notera:

$$
q\left(e, P_{2}^{E}\right)=\int_{q^{I}}^{\bar{q}} q f\left(q / P_{2}^{E}, e\right) d q
$$

On cherche un équilibre bayésien parfait de ce jeu.

Définition 1 Un équilibre bayésien parfait est un vecteur de stratégie $e^{*}(q),\left(P_{2}^{E}(q)\right)^{*},\left(P_{2}^{I}\right)^{*},\left(P_{3}^{E}\right)^{*},\left(P_{3}^{I}\right)^{*}$ et de croyances $f\left(q \mid e^{*},\left(P_{2}^{E}\right)^{*}\right)$ tel que :

(P1)-Firme $E: e^{*},\left(P_{2}^{E}\right)^{*},\left(P_{3}^{E}\right)^{*} \in \underset{P_{2}}{\arg \max } \Pi^{E}\left(e, P_{2}^{E}, q\left(e^{*},\left(P_{2}^{E}\right)^{*}\right)\right)$

$$
+\Pi^{E}\left(e, P_{3}^{E}, q\right)
$$

Firme $I:\left(P_{2}^{I}\right)^{*},\left(P_{3}^{I}\right)^{*} \in \underset{P_{2}^{I}, P_{3}^{I}}{\arg \max } \int_{q^{I}}^{\bar{q}} f\left(q \mid e^{*},\left(P_{2}^{E}\right)^{*}\right) \Pi^{I}\left(e^{*}, P_{2}^{I}, q^{I}\right) d q$

$$
+\Pi^{I}\left(e^{*}, P_{3}^{I}, q^{I}\right)
$$

(P2)- les croyances $f\left(q \mid e^{*},\left(P_{2}^{E}\right)^{*}\right)$ sont révisées selon une règle de Bayes si la localisation $e^{*}$ et les prix sont observés et indéterminées sinon.

En troisième étape, les consommateurs découvrent la qualité du bien proposé par l'entreprise. $\left(P_{3}^{E}\right)^{*}$ et $\left(P_{3}^{I}\right)^{*}$ sont donc les prix d'équilibre du jeu à une période en information complète déterminé à la section précédente. $\Pi^{i}\left(e, P_{3}^{i}, q\right)$ est alors le profit du jeu en information complète à une période. On note désormais $\Pi()$ le profit de la seule firme $E$. Notons que ce profit est une fonction croissante de la qualité $q$. Une firme ayant découvert une qualité

7 Dorénavant, l'indice désignera la période et non plus le marché. Aucune contusion n'est possible puisqu'il s'agit de 2 ou 3 et non $N$ et $S$. 
$q$ n'a donc aucun intérêt à choisir une qualité inférieure. Par conséquent, les croyances a priori des consommateurs sont données par la fonction $f$.

La firme entrante peut signaler la qualité de son produit par le prix et la localisation choisis. l'entreprise entrante dispose donc d'une infinité d'instruments, il peut ainsi a-priori exister un équilibre séparateur. On montre toutefois le lemme suivant.

Lemme 1 Le prix fixé par la firme $E$ ne lui permet pas de signaler la qualité de son bien. Il n'existe donc pas d'équilibre parfaitement séparateur.

Preuve Annexe A.

Un tel résultat se comprend aisément. Le prix ne constitue pas un engagement crédible : la firme entrante peut réviser son prix d'une période sur l'autre. Une firme a donc toujours intérêt à « imiter » le prix signalant la meilleure qualité.

On se concentre donc sur les seuls équilibres semi-séparateur, pour lequel seule la localisation sert de signal, et mélangeant. Examinons alors la localisation de la firme $E$.

\subsection{Localisation d'équilibre}

On montre que le modèle satisfait la condition de Spence Mirrlees.

Lemme 1 Quel que soit $\lambda$,

$$
\frac{\partial \Pi\left(S, P_{2}^{E}, q(.)+\Pi\left(S,\left(P_{3}^{E}\right)^{*}, q\right)\right.}{\partial q} \leqslant \frac{\partial \Pi\left(N, P_{2}^{E}, q(.)\right)+\Pi\left(N,\left(P_{3}^{E}\right)^{*}, q\right)}{\partial q}
$$

\section{Preuve Annexe A.}

口

Cela signifie que plus la qualité de la firme entrante est faible, plus une implantation dans la région Nord est pénalisante. En effet, en se localisant en $N$, une firme de bonne qualité craint peu la concurrence de la firme installée et a par ailleurs accès au marché de la région Sud. Au contraire, une firme vendant un produit de mauvaise qualité est pénalisée par la forte concurrence de la firme installée. Le coût d'une telle localisation pour la firme entrante provient de l'impossibilité de se relocaliser en troisième période ${ }^{8}$.

Ainsi, s'il existe une qualité $\tilde{q}$ pour laquelle la firme entrante est indifférente aux deux localisations, une firme dont la qualité est supérieure s'implantera en $N$ et une firme dont la qualité est inférieure se localisera dans la région $S$.

Par conséquent, on ne cherche que des équilibres semi-séparateurs ou mélangeants. Un équilibre semi-séparateur se caractérise par le fait que si

8 L'introduction d'un paramètre d'actualisation inférieur à 1 ne modifie pas qualitativement ce résultat; il reviendrait à amoindrir le coût de l'agglomération pour une entreprise. 
la qualité de l'entreprise innovante appartient à l'intervalle $[\tilde{q}, \bar{q}]$, elle se localise dans une région Nord et dans le cas contraire, elle s'implante dans la région Sud. Au contraire dans un équilibre mélangeant, la firme entrante s'implante dans la même région quelle que soit la qualité du bien proposé.

On obtient alors le résultat suivant.

Lemme $3 n$ existe un seuil $\overline{q^{m}}(\lambda)$ tel que:

(i) Si la qualité moyenne $q^{m}$ est élevée $\left(q^{m}>\overline{q^{m}}(\lambda)\right)$, il existe toujours deux équilibres:

- Un équilibre mélangeant dans lequel la firme entrante se localise toujours dans la région Nord.

- Un équilibre mélangeant dans lequel la firme entrante se localise toujours dans la région Sud.

(ii) Si la qualité moyenne $q^{m}$ est faible $\left(q^{m}<\overline{q^{m}}(\lambda)\right)$, il existe toujours deux équilibres:

- Un équilibre semi-séparateur dans lequel si $q$ est supérieur $\grave{a} \tilde{q}(\lambda)$, l'entreprise entrante se localise dans la région Nord. Dans les autres cas $(q<\tilde{q}(\lambda))$, la firme se localise dans la région Sud.

- Un équilibre mélangeant dans lequel pour tout q la firme se localise dans la région Sud.

\section{Preuve}

L'absence d'équilibre parfaitement séparateur permet de simplifier les notations : la qualité anticipée $q($.$) ne dépend que de la localisation e$ de la firme entrante. Cette qualité est donc soit $q(N)$ soit $q(S)$. Notons $Q(e)$ l'ensemble des qualités de la firme entrante lorsque cette dernière se localise en e. Dans ce cas :

$$
q(e)=\int_{q^{I}}^{\bar{q}} q f(q / e) d q
$$

où :

$$
\begin{aligned}
& f(q \mid N)= \begin{cases}\frac{f(q)}{\int_{Q(N)} f(s) d s} & \text { si } q \in Q(N) \\
0 & \text { sinon }\end{cases} \\
& f(q \mid S)= \begin{cases}\frac{f(q)}{\int_{Q(S)} f(s) d s} & \text { si } q \in Q(S) \\
0 & \text { sinon }\end{cases}
\end{aligned}
$$

Il existe un seul équilibre mélangeant en prix. Cet équilibre en prix $\left(P_{2}^{E}\right)^{*}$ et $\left(P_{2}^{I}\right)^{*}$ est l'équilibre en prix en information parfaite lorsque la qualité anticipée par les consommateurs est $q(e)$.

Lorsque la firme entrante est localisée en $N$ :

$$
\begin{aligned}
& \left(P_{2}^{E}\right)^{*}=\operatorname{Min}\left(\left(q(N)-q^{I}\right)+t, q(N)\right) \\
& \left(P_{2}^{I}\right)^{*}=t
\end{aligned}
$$


avec $t=0$ s'il s'agit du marché Nord et $t=\lambda$ s'il s'agit du marché Sud.

Lorsque la firme entrante est localisée en $S$, sur le marché Sud :

$$
\begin{aligned}
& \left(P_{2}^{E}\right)^{*}= \begin{cases}q(S) & \text { si } \lambda \geqslant q^{I} \\
\left(q(S)-q^{I}\right)+\lambda & \text { si } \lambda \leqslant q^{I}\end{cases} \\
& \left(P_{2}^{I}\right)^{*}=\lambda
\end{aligned}
$$

Sur le marché Nord:

$$
\begin{aligned}
& \left(P_{2}^{E}\right)^{*}=\operatorname{Min}\left(\left(q(S)-q^{I}\right), \lambda\right) \\
& \left(P_{2}^{I}\right)^{*}=0
\end{aligned}
$$

Les croyances :

$$
\begin{aligned}
f\left(q(e) \mid\left(P_{2}^{E}\right)^{*}, e\right) & =1 \\
f\left(q \mid P_{2}^{E}, e\right) & =0 \quad \forall P_{2}^{E} \neq\left(P_{2}^{E}\right)^{*}
\end{aligned}
$$

(a) Pour montrer assurer l'existence d'un équilibre semi-séparateur en localisation, il doit exister une firme indifférente entre une localisation en $N$ ou en $S$. Si elle existe, cette qualité $\bar{q}$ vérifie l'égalité suivante (on omettra désormais le prix dans les expressions des profits):

$$
\Pi(N, q(N))+\Pi(N, \tilde{q})=\Pi(S, q(S))+\Pi(S, \tilde{q})
$$

Grâce au lemme 2, si une qualité $\tilde{q}$ s'implante en $N$, une firme de qualité supérieure s'implantera aussi en $N$. Par conséquent :

La firme de meilleure qualité a bien intérêt à se signaler :

$$
\Pi(N, \bar{q})+\Pi(N, \bar{q}) \geqslant \Pi\left(S, q^{m}\right)+\Pi(S, \bar{q})
$$

En effet dans le cas où seule la qualité $\bar{q}$ s'implante en $N, q(N)=\bar{q}$ et donc $q(S)=q^{m}$.

La fonction $\left(\Pi(N, \bar{q})+\Pi(N, \bar{q})-\Pi\left(S, q^{m}\right)-\Pi(S, \bar{q})\right)$ décroît avec $q^{m}$ et $\lambda$. Par conséquent l'inégalité $(24)$ est vraie si $q^{m}<\underline{q^{m}}(\lambda)$ où $\underline{q}^{m}(\lambda)$ est une fonction décroissante de $\lambda$.

Notons que si $\lambda<\bar{q}-q^{I}, \underline{q^{m}}(\lambda)=\bar{q}$ de sorte que (24) est toujours vraie. En effet :

$$
\Pi(N, \bar{q})+\Pi(N, \bar{q})=\Pi(S, \bar{q})+\Pi(N, \bar{q})>\Pi\left(S, q^{m}\right)+\Pi(S, \bar{q})
$$

De plus, si $\lambda \geqslant \bar{q}, \underline{q^{m}}(\lambda)=\bar{q}-2 q^{I}$.

Par ailleurs, la firme $q^{\prime}$ n'a pas intérêt à dévier de $S$ vers $N$ si :

$$
\Pi\left(N, q^{m}\right)+\Pi\left(N, q^{I}\right) \leqslant \Pi\left(S, q^{I}\right)+\Pi\left(S, q^{I}\right)
$$


On clémontre dans l'annexe $B$ que cette égalité est, vérifiée si :

$$
q^{m}<\overline{q^{m}}(\lambda)
$$

où $\overline{q^{m}}(\lambda)$ est une fonction croissante de $\lambda$.

Notons que si $\lambda \geqslant \bar{q}, \overline{q^{m}}(\lambda)=2 q^{I}$.

Par conséquent, si $\bar{q}>4 q^{I}, \overline{q^{m}}(\lambda) \leqslant \underline{q^{m}}(\lambda)$.

En revanche, si $\bar{q}<4 q^{I}, \overline{q^{m}}(\lambda) \leqslant \underline{q^{m}}(\lambda)$ si et seulement si $\lambda<\hat{\lambda}$.

Par ailleurs le lemme 2 assure la croissance et la monotonie en $\tilde{q}$ de la fonction :

$$
(\Pi(N, q(N))+\Pi(N, \tilde{q}))-(\Pi(S, q(S))+\Pi(S, \tilde{q}))
$$

Par conséquent, $\tilde{q}$ existe et est unique dès que $q^{m}<\overline{q^{m}}(\lambda)$.

(b) Il existe un équilibre mélangeant dans lequel firme entrante se localise en $S$ quelle que soit la qualité $q$ si elle n'a jamais intérêt à dévier vers la région $N$. Le lemme 2 montre que c'est la firme de qualité $\bar{q}$ qui est la plus incitée à quitter $S$ pour se localiser en $N$. Les croyances hors équilibre $f(q \mid N)$ doivent donc vérifier :

$$
\Pi(N, q(N))+\Pi(N, \bar{q})<\Pi\left(S, q^{m}\right)+\Pi(S, \bar{q})
$$

C'est le cas si $q(N)<q^{m}$.

(c) De la même façon, il existe un équilibre mélangeant en $N$ si lorsque la firme entrante s'implante en $N$ quelle que soit sa qualité, elle n'a intérêt à clévier. Le lemme 1 montre que c'est la firme $q^{I}$ qui a le plus intérêt à dévier. Cette dernière ne dévie effectivement pas si les croyances hors équilibre $f(q \mid N)$ vérifient :

$$
\Pi\left(N, q^{m}\right)+\Pi\left(N, q^{l}\right)>\Pi(S, q(S))+\Pi\left(S, q^{I}\right)
$$

Si $q^{m}<\overline{q^{m}}(\lambda, q)$, on ne peut trouver de croyances hors équilibre permettant de vérifier l'inégalité précédente. En effet, même si la firme de qualité $q^{I}$ est repérée comme telle dans la région $S$, cette dernière préfère malgré tout se localiser en $S$.

En revanche, si $q^{m}>\overline{q^{m}}(\lambda)$, il existe $q(S)$ qui rend l'inégalité précédente vérifiée avec $q(S)<q^{m}$.

La coexistence de plusieurs équilibres impose de retenir un critère de raffinement permettant d'en sélectionner un seul, mélangeant ou semiséparateur, selon les paramètres. Seul le critère de Umbhauer (1994) permet en définitive de ne conserver qu'un équilibre selon la valeur de $q^{m}$. Le critère de raffinement est le suivant ${ }^{9}$.

9 Les autres critères comme le critère intuitif de Chow et Kreps sont insulfisants. 
Définition 2 Soient deux équilibres $G^{*}$ et $G^{* *}$ et $e^{*}(q)$ et $e^{* *}(q)$ les localisations d'équilibre si $E$ est de type $q$ respectivement dans l'équilibre $G^{*}$ et $G^{* *}$.

Un équilibre $G^{*}$ " détruit " $G^{* *}$ s'il existe un sous ensemble de qualités $Q$ tel que :

(i) $\Pi^{*}(q) \geqslant \Pi^{* *}(q)$ pour tout $q \in Q$

$\Pi^{*}(q)<\Pi^{* *}(q)$ pour tout $q \in\left[q^{I}, \bar{q}\right] \backslash Q$

(ii) Il existe certaines qualités $q$ appartenant à $Q$ pour lesquelles les croyances $f^{* *}\left(q \mid\right.$.) formées hors équilibre dans le cas où l'équilibre $G^{* *}$ se réalise sont telles que:

$$
f^{* *}\left(q \mid e^{*}(q)\right) \neq \frac{f(q)}{\int_{Q} f(s) d s}
$$

Ce critère de raffinement revient à examiner si les croyances hors équilibre qui soutiennent un équilibre sont " raisonnables " ${ }^{10}$.

(i) On considère que les seuls types qui sont susceptibles de dévier et de choisir une localisation $e$ sont ceux qui gagneraient (ou ne perdraient pas) à ce que l'équilibre pour lequel existe effectivement la localisation $e$ se réalise (sous ensemble $Q$ défini précédemment). On doit recentrer les croyances concernant la localisation hors équilibre $e$ sur ce sous-ensemble.

(ii) Si ce recentrage ne permet plus de soutenir l'équilibre dans lequel la localisation $e$ est une localisation hors équilibre, les croyances ne sont pas jugées « raisonnables » et l'équilibre est rejeté.

À l'aide de ce critère de raffinement on parvient à éliminer l'équilibre mélangeant dans lequel les entreprises se dispersent et à ne retenir que l'équilibre semi-séparateur ou l'équilibre mélangeant dans lequel les deux firmes s'agglomèrent. On obtient alors la proposition suivante.

Proposition 2 (i) Lorsque la firme installée est localisée dans la région Nord, la firme entrante s'implante dans cette même région si la qualité de son produit est supérieure $\dot{a} \tilde{q}\left(\lambda, q^{m}\right)$. Dans le cas contraire, elle se localise dans la région Sud.

(ii) L'agglomération des entreprises se produit pour un intervalle de qualité plus large en information incomplète qu'en information parfaite: $\tilde{q}\left(\lambda, q^{m}\right)<\hat{q}(\lambda)$

(iii) Si la qualité moyenne espérée par les consommateurs est élevée $\left(q^{m}>\overline{q^{m}}(\lambda)\right)$, la qualité seuil $\tilde{q}\left(\lambda, q^{m}\right)$ est égale à $q^{I}$ de sorte que quelle que soit sa qualité, la firme entrante se localise dans la région Nord.

10 Ce critère est très proche de celui de Mailath, Okuno-Fujiwara et Postlewaite (1993) adapté par Boyer, Laffont, Mahenc et Moreaux (1995), dans un cadre proche. Toutefois, ce dernier critère impose que $\mathrm{II}^{*}(q)>\Pi^{* *}(q)$ pour un $q \in Q$. Or pour ne retenir qu'un des deux équilibres mélangeants, cette condition est trop forte. 


\section{Preuve}

(i) Considérons d'abord le cas où $q^{m}<\overline{q^{m}}(\lambda)$, c'est à dire le cas où un équilibre semi-séparateur $G^{*}$ et un équilibre mélangeant $G^{* *}$ coexistent. Montrons alors que $G^{*}$ détruit $G^{* *}$ au sens du critère de Umbhauer (1994). On reprend successivement les deux points du critère de raffinement défini précédemment :

(a) Construisons l'ensemble $Q$ défini précédemment. On montre aisément qu'il existe une firme de qualité $q^{l}$ dont les gains selon l'équilibre retenu sont identiques. La qualité $q^{l}$ vérifie :

$$
\Pi(N, q(N))+\Pi\left(N, q^{l}\right)=\Pi\left(S, q^{m}\right)+\Pi\left(S, q^{l}\right)
$$

On est certain de l'existence de $q^{l}$ pour deux raisons. D'une part la qualité $\bar{q}$ préfère strictement l'équilibre semi-séparateur à l'équilibre mélangeant (cf équation (24)) et la qualité $q^{I}$ préfère strictement l'équilibre mélangeant à l'équilibre séparateur. D'autre part, la fonction $\Pi(N, q(N))+$ $\Pi(N, q)-\Pi\left(S, q^{m}\right)-\Pi(S, q)$ est monotone croissante en $q$ et continue en $q$ (lemme 2). $q^{l}$ existe et est unique.

Il existe donc un sous-ensemble de variétés $Q\left(Q=\left[q^{l}, \bar{q}\right]\right)$ qui préfèrent la réalisation de l'équilibre semi-séparateur à la réalisation de l'équilibre mélangeant.

Représentons graphiquement (figure 5) les profits d'une entreprise de qualité $q$ réalisés en $N\left(\Pi\left(N, q^{*}(N)\right)+\Pi(N, q)\right.$, équilibre semi-séparateur) et en $S\left(\Pi\left(S, q^{m}\right)+\Pi(S, q)\right.$, équilibre mélangeant $)$.

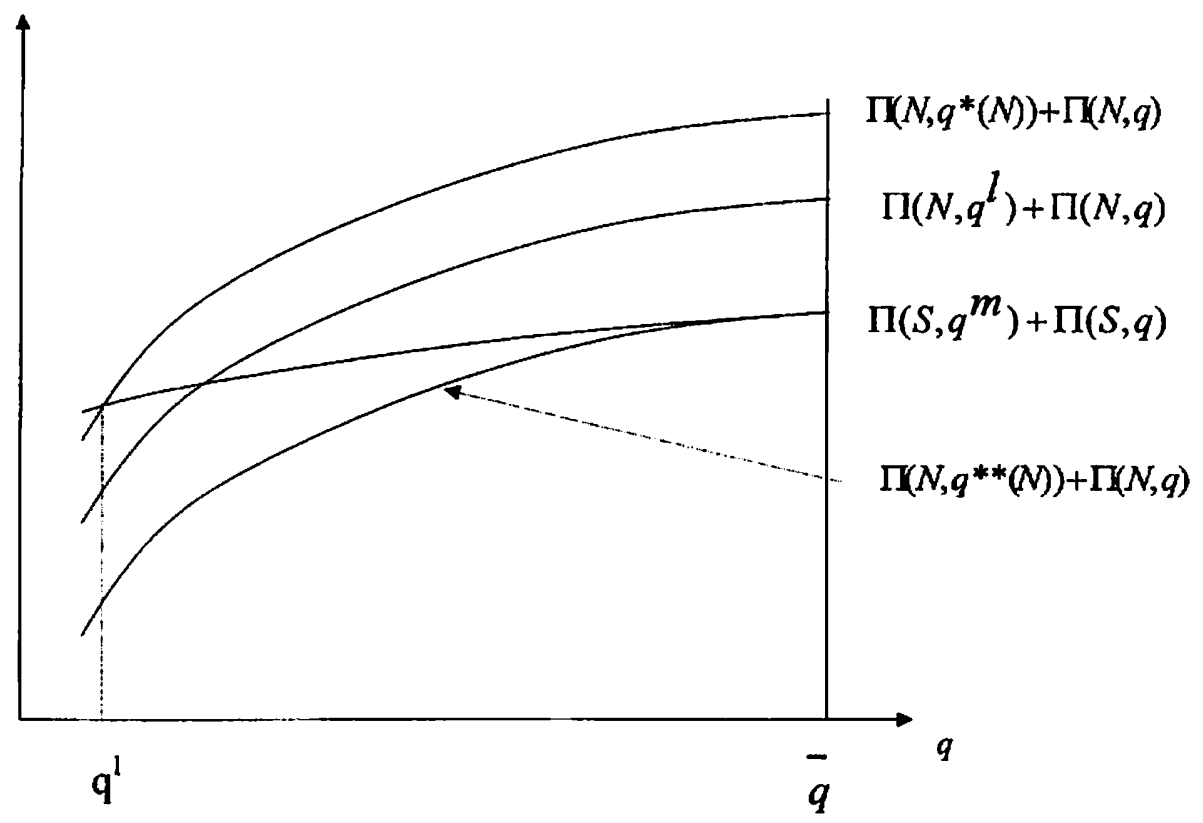

Figure 5 
Par définition, la qualité $q^{l}$ est donc à l'intersection de la courbe $\Pi(N, q(N))+\Pi\left(N, q^{l}\right)$ et de la courbe $\Pi\left(S, q^{m}\right)+\Pi\left(S, q^{l}\right)$.

(b) Vérifions que les croyances hors-équilibre ne sont pas raisonnables. Les croyances hors équilibre permettant d'assurer l'existence de l'équilibre mélangeant $G^{* *}, f^{* *}(q \mid N)$, sont telles que la firme $\bar{q}$ ne souhaite jamais dévier en choisissant la localisation $N$. Ces croyances doivent donc être telles que la courbe $\Pi\left(N, q^{* *}(N)\right)+\Pi(N, q)$ soit toujours en dessous de la courbe $\Pi\left(S, q^{m}\right)+\Pi(S, q)$.

Il faut montrer à présent que ces croyances hors équilibre $f^{* *}(q \mid N)$ ne peuvent être toutes égales à $\frac{f(q)}{J_{Q} f(s) S s}$. On va simplement montrer pour cela que $q^{l}>q^{* *}(N)$ c'est à dire qu'une entreprise de qualité perçue $q^{l}$ est strictement incitée à quitter la région pour s'agglomérer (dévier de $S$ vers $N)$.

Pour que ce soit le cas, il suffit que la courbe $\Pi\left(N, q^{l}\right)+\Pi(N, q)$ soit au dessus de la courbe $\Pi\left(N, q^{* *}(N)\right)+\Pi(N, q)$. Il existe une qualité $q$ indifférente entre $N$ si sa qualité perçue est $q^{l}$ ou $S$ :

$$
\Pi\left(N, q^{l}\right)+\Pi(N, q)=\Pi\left(S, q^{m}\right)+\Pi(S, q)
$$

En effet, on sait que $q^{l}$ existe quel que soit $q^{*}(N)$. Or si $q^{*}(N)=q^{l}$, il existe donc $q$ tel que :

$$
\Pi\left(N, q^{l}\right)+\Pi(N, q)=\Pi\left(S, q^{m}\right)+\Pi(S, q)
$$

En d'autres termes, il existe donc toujours une intersection entre la courbe $\Pi\left(N, q^{l}\right)+\Pi(N, q)$ et la courbe $\Pi\left(S, q^{m}\right)+\Pi(S, q)$. Cela assure finalement que $q^{* *}(N)<q^{l}$

Lorsque $q^{m}>\overline{q^{m}}$, on montre de la même façon que l'équilibre mélangeant où $e^{*}=N$ « détruit » l'équilibre mélangeant où $e^{*}=S$.

(ii) Par définition, $2 \Pi(N, \hat{q}(\lambda))=2 \Pi(S, \hat{q}(\lambda))$. La firme dont la qualité est $\hat{q}(\lambda)$ ne peut être indifférente entre les deux localisations. En effet, en s'implantant en $N$, la qualité perçue par les consommateurs est supérieure à $\hat{q}(\lambda)$. Une firme de qualité $\hat{q}(\lambda)$ est donc incitée à se localiser en $N$ : $\tilde{q}(\lambda)<\hat{q}(\lambda)$. suivant.

En déterminant sa localisation, le nouvel entrant fait face à l'arbitrage

S'il s'implante dans la même région que la firme installée, il profite du fait qu'il s'agit de la région qui pénalise une entreprise peu innovante (lemme 2) de sorte que les consommateurs anticipent qu'une firme s'y localisant produit un bien de qualité élevée.

Au contraire, s'il s'implante dans l'autre région, il évite la concurrence frontale de la firme installée mais pâtit d'une mauvaise anticipation de la qualité de la part des consommateurs. 
Les termes de cet arbitrage nous permet de comprendre les trois points de cette proposition.

(i) Si la qualité moyenne attendue ex-ante est élevée ( $q^{m}$ élevé), seul l'équilibre mélangeant dans lequel les deux entreprises sont agglomérées subsiste. Ce résultat se comprend bien : si les consommateurs s'attendent à ce que le bien de la firme entrante soit de qualité élevée, la firme entrante cherche à profiter de ces croyances favorables en choisissant une localisation identique à celle de la firme installée : le coût d'agglomération spatiale (la concurrence) est toujours compensé par l'avantage qu'elle confère (qualité anticipée élevée). Le coût induit par l'agglomération spatiale est dû à l'impossibilité de se relocaliser en troisième étape. Par ailleurs, un paramètre d'actualisation inférieur à 1 viendrait réduire un tel coût et par conséquent viendrait accroître l'incitation pour une firme à se localiser dans la région Nord.

Lorsque $q^{\prime \prime}$ est faible, on ne retient bien que l'équilibre semi-séparateur : le coût de la polarisation géographique (concurrence) finit pour un produit peu innovant $\left(q^{E}<\tilde{q}\right)$ par l'emporter sur un avantage insuffisant (qualité anticipée plus élevée pour $N$ que pour $S$ mais insuffisamment).

(ii) En information parfaite, la firme entrante est au mieux indifférente entre les deux localisations. Par conséquent, dans ce dernier cas et en présence d'asymétrie d'information, la firme choisit de se localiser dans la région Nord afin de signaler une qualité élevée.

L'agglomération spatiale est un moyen pour les firmes de signaler aux consommateurs l'aspect innovant de leur produit. La crédibilité de ce signal repose sur l'impossibilité pour une firme de se relocaliser lorsque les consommateurs prennent connaissance de la véritable qualité du produit. Seule l'asymétrie d'information permet ici l'émergence de la polarisation géographique des entreprises qui ne se produit pas en information parfaite. En effet, en information parfaite, la firme entrante est au mieux indifférente entre les deux localisations. Au contraire, en information incomplète, elle choisit de se localiser dans la région Nord et signale ainsi une qualité élevée.

Cette forte agglomération spatiale est un résultat cohérent avec les études empiriques sur la localisation des firmes, et notamment des firmes innovantes, qui mettent en évidence une forte tendance à la polarisation de l'activité. On montre également que la concurrence stimule l'innovation (Audretsch et Feldman (1999) ou Jacobs (1984)). Les meilleures firmes choisissent de développer leur innovation dans la région où la concurrence est la plus forte pour signaler aux consommateurs leur valeur. On parvient ainsi à expliquer le développement de pôles d'innovation. Par exemple le développement économique de certaines villes du sud de l'Italie repose toujours sur la réussite préalable d'une entreprise. Une fois le succès avéré, de nouvelles firmes se développent à proximité de la première. Les districts industriels spécialisés dans l'industrie du meuble ou de la chaussure se sont développés 
de cette façon. ${ }^{11}$ Porter (1998) montre aussi que de nombreux pôles géographiques ont pour origine le développement d'une firme pionnière : la spécialisation de Minneapolis dans la fabrication d'appareils médicaux tient à la présence initiale d'une firme, Medtronic.

On peut expliquer également les nombreuses déconvenues qui ont suivi des programmes de développement de régions défavorisées comme l'Italie du Sud ou l'Andalousie. Des efforts considérables de modernisation des infrastructures ont été menés dans la région des Pouilles au sud de l'Italie (Lieutaud (1991)). Il semble toutefois que les résultats soient décevants : le taux de chômage reste très élevé et le taux de migration s'est même accru. Jacobs (1984) souligne que la présence dans une région d'un embryon d'industrie est un critère décisif pour la réussite d'une politique de développement économique.

En effet, le niveau initial de développement est ici déterminant : la présence de firmes concurrentes dans une région loin de dissuader d'éventuels entrants va au contraire leur servir de «faire-valoir » et les inciter à se localiser dans cette région. La présence d'une entreprise concurrente dans la région Nord constitue pas un handicap pour séduire les entreprises innovantes mais au contraire un avantage. On donne ainsi une nouvelle justification à l'importance de l'histoire dans le choix de localisation des entreprises (Rauch (1993) et Krugman (1991)). Une région devient d'autant plus attractive que des firmes s'y sont auparavant implantées. Un phénomène cumulatif s'enclenche à l'origine duquel se trouve ici un moyen de signaler la différenciation des biens.

Si le progrès technique se caractérise par une augmentation de la qualité des biens de la même façon que dans Aghion et Howitt (1992), les asymétries d'informations sont sources de développement inégal. En effet, lorsque le progrès technique moyen est élevé, la firme innovante se localise systématiquement dans la région qui profitait déjà de la présence de la firme précédemment leader technologique (point (iii) de la proposition).

Remarquons en dernier lieu que, dans ce modèle, la firme entrante met à profit le choix de localisation pour signaler la qualité du bien aux seuls consomateurs. Il est vraisemblable que si la firme devait emprunter une certaine somme auprès d'une banque, la localisation lui permette de signaler la qualité du bien au prêteur ${ }^{12}$.

On examine à présent l'impact d'une baisse des coûts de transport sur la localisation de la firme entrante.

\footnotetext{
11 Entretien avec Luca Meldosi, professeur à l'Université de Naples dans Le Monde du mardi 24 novembre 1998.

12 Le développement d'un tel mécanisme exige l'introduction d'un emprunt ainsi que d'un secteur bancaire.
} 


\section{$5 \quad$ Impact du désenclavement}

Cette section étudie l'impact d'une modernisation de l'infrastructure de transport se traduisant par une diminution du coût de transport $(\lambda)$. On examine l'influence de la baisse du coût de transport sur la localisation de la firme entrante ainsi que sur le bien-être de l'économie. On néglige le coût de la modernisation de l'infrastructure de transport. On se concentre uniquement sur l'éventuel seul bénéfice de cette modernisation.

\subsection{Coût de transport et localisation}

La baisse du coût de transport inter-régional rapproche la région $S$ de la région $N$. En se localisant en $N$ la firme de qualité $\tilde{q}(\lambda)$ profite d'un meilleur accès à la région $S$ :

$$
\frac{\partial(\Pi(N, q(N))+\Pi(N, \tilde{q}))}{\partial \lambda} \leqslant 0
$$

Par ailleurs, la baisse de $\lambda$ se traduit par une intensification de la concurrence au sein de la région $S$.

$$
\frac{\partial(\Pi(S, q(S))+\Pi(S, \tilde{q}))}{\partial \lambda} \geqslant 0
$$

La région Sud perd donc tout attrait : elle cesse d'être une région à l'abri de toute concurrence et reste associée à un produit de qualité médiocre.

On montre alors la proposition suivante.

Proposition 3 (i) Une baisse du coût de transport inter-régional ( $\lambda$ ) se traduit par une diminution du seuil $\tilde{q}(\lambda)$ renforçant ainsi les chances de polarisation spatiale.

(ii) Il existe un niveau du coût de transport $(\bar{\lambda})$ en deçà duquel la firme entrante se localise toujours dans la région Nord.

\section{Preuve} dans les cas 2 et 3 .

(i) $\frac{\partial \Pi(S, q(S))}{\partial \lambda}+\frac{\partial \Pi(S, \tilde{q})}{\partial \lambda}=2$ dans le cas 1 et $\frac{\partial \Pi(S, q(S))}{\partial \lambda}+\frac{\partial \Pi(S, \bar{q})}{\partial \lambda}=0$ ou -2 dans les cas 2 et 3 .

$\frac{\partial \Pi(N, q(N))}{\partial \lambda}+\frac{\partial \Pi(N, q)}{\partial \lambda}=-2$ dans le cas 1 et $\frac{\partial \Pi(N, q(N))}{\partial \lambda}+\frac{\partial \Pi(N, q)}{\partial \lambda}=0,-1$

Donc : $\frac{\partial \Pi(S, q(S))}{\partial \lambda}+\frac{\partial \Pi(S, \tilde{q})}{\partial \lambda}-\frac{\partial \Pi(N, q(N))}{\partial \lambda}+\frac{\partial \Pi(N, q)}{\partial \lambda} \geqslant 0$

Montrons que $\tilde{q}(s, \lambda+d \lambda)<\tilde{q}(s, \lambda)$ avec $d \lambda<0$.

Du fait de l'inégalité précédente si le coût de transport devient $\lambda+d \lambda$, on $\mathbf{a}$ : 
$\Pi(N, q(N))+\Pi(N, \tilde{q}(\lambda))>\Pi(S, q(S))+\Pi(S, \tilde{q}(\lambda))$. La qualité $\tilde{q}(\lambda)$ bascule vers la région Nord. Par conséquent, $\tilde{q}(\lambda)>\tilde{q}(\lambda+d \lambda)$

(ii) Le seuil $\bar{\lambda}$ vérifie $q^{m}=\overline{q^{m}}(\bar{\lambda})$. Les expressions de $\overline{q^{m}}(\lambda)$ déterminées dans l'annexe $B$ assurent de l'existence d'un tel seuil. Par ailleurs, $\overline{q^{m}}(\lambda)$ est une fonction croissante de $\lambda$. Donc, pour tout $\lambda<\bar{\lambda}$, on a également $q^{m}>\overline{q^{m}}$.

La construction de l'infrastructure inter-régionale profite indéniablement à la région Nord et favorise l'agglomération des entreprises. Par ailleurs si la baisse du coût de transport inter-régional est suffisamment importante, le nouvel entrant se localise en définitive toujours dans le Nord. Cette proposition souligne qu'une baisse des coûts de transport inter-régionaux peut se traduire par l'émergence d'un seul pôle d'entreprises innovantes. En information parfaite, au contraire, un nouvel entrant de faible qualité se localisait dans la région Sud.

La section suivante étudie l'impact de ces diminutions sur le bienêtre pour déterminer s'il est préférable de lutter contre la polarisation des entreprises en favorisant les infrastructures intra-régionales.

\subsection{Désenclavement et bien-être}

Une autorité supra-régionale envisage d'investir dans une infrastructure de transport. Cette section mesure et compare l'impact d'une diminution du coût de transport inter-régional ou intra-régional sur le bien-être total de l'économie.

On montre préalablement que les localisations d'équilibre se traduisent par une sur-agglomération des firmes au regard du bien-être de l'économie.

\subsubsection{Localisation et bien-être en information complète}

Déterminons, à l'équilibre en prix, la localisation optimale de la firme entrante dont la qualité du bien est égale à $q$. On distingue pour cela deux cas.

(i) Si la firme entrante ne peut servir le marché de la région dans laquelle elle n'est pas implantée $(q<\lambda)$, il est socialement préférable que les deux firmes ne se localisent pas dans la même région. En effet, si les firmes s'agglomèrent, seuls les consommateurs de la région Nord profite du bien : le bien-être noté $W^{A}(q, \lambda)$ est égal à $q$. En revanche, si les deux firmes se dispersent, les consommateurs de la région Nord profitent du bien de la firme installée et les consommateurs du Sud consomment le nouveau produit : le bien-être $W^{D}(q)$ est dans ce cas égal à $q+q^{J}$.

(ii) Si la firme entrante peut servir le marché Sud en étant localisée dans le Nord mais ne peut toutefois pas servir le marché Nord en étant localisée dans la région Sud $\left(\lambda<q<\lambda+q^{I}\right)$, la localisation optimale dépend du résultat de l'arbitrage suivant. Lorsque les deux firmes sont agglomérées 
dans la région Nord, les consommateurs des deux régions profitent du nouveau bien qui doit toutefois être transporté d'une région à l'autre. Le bienêtre est alors égal à $W^{A}=2 q-\lambda$. Lorsque la firme entrante est localisée en $S$, seuls les consommateurs de cette région consomment le nouveau bien: $W^{D}=q+q^{I}$. L'agglomération spatiale est donc socialement souhaitable si $q \geqslant q^{I}+\lambda$ : le différentiel de qualité doit être supérieur au coût de transport inter-régional.

En information parfaite la localisation de la firme entrante est donc optimale $\left(\hat{q}=q^{I}+\lambda\right)$. Un tel résultat n'est pas surprenant : la spécification des préférences des consommateurs et la politique de prix rendu permet à la firme entrante de s'approprier tout le surplus des consommateurs.

Par conséquent, la présence d'asymétrie d'informations se traduit par trop d'agglomération spatiale puisque $\bar{q}$ est inférieur à $\hat{q}: W^{A}(\tilde{q})<W^{D}(\tilde{q})$. La détermination des bien-êtres régionaux permet de déterminer la région qui supporte cette inefficacité.

\subsubsection{Localisation et bien-êtres régionaux}

La localisation des entreprises a un impact déterminant sur le bien-être de chacune des deux régions. Examinons le bien-être des régions Sud et Nord.

On note $W S^{D}$ (respectivement $W S^{A}$ ) le surplus de la région Sud lorsque les fimes sont dispersées (respectivement agglomérées). On on obtient :

$$
W S^{D}(q, \lambda)=q>W S^{A}(q, \lambda)= \begin{cases}0 & \text { si } \lambda>q^{I} \\ q^{I}-\lambda & \text { si } \lambda<q^{I}\end{cases}
$$

L'agglomération des firmes dans le Nord pénalise la région Sud. Par ailleurs, lorsque les entreprises s'agglomèrent dans la région Nord, une baisse du coût de transport est susceptible de profiter à la région Sud. En effet, dans ce cas, lorsque la firme entrante est en concurrence avec la firme installée (c'est le cas dès que $\lambda<q^{I}$ ), les consommateurs de la région Sud supportent le coût de transport nécessaire pour acheminer le bien et répercuté par la firme.

Au contraire, le Nord profite toujours de l'agglomération des firmes. En effet, le bien-être de la région Nord est égal à :

$$
W N^{D}=q^{I}<W N^{A}= \begin{cases}q+q-\lambda & \text { si } \lambda>q^{I} \\ q+\left(q-q^{I}\right) & \text { si } \lambda<q^{I}\end{cases}
$$

Par conséquent, la sur-agglomération des firmes due à l'asymétrie d'information pénalise la région Sud au profit de la région Nord.

Nous pouvons à présent nous concentrer sur l'impact d'une diminution des coûts de transport en présence d'asymétrie d'informations. 


\subsubsection{Localisation, politique d'infrastructure et bien-être en information incomplète}

Les autorités supra-régionales se concentrent sur le bien-être espéré ex-ante.

Ex-post, si la qualité du produit de la firme entrante est suffisamment élevée $(q>\tilde{q})$, les deux entreprises sont spatialement agglomérées dans la région $N$ alors que si cette qualité est plus faible, la firme entrante se localise dans le sud.

Ex-ante, le bien-être espéré $W(\lambda)$ est donc la somme pondérée des bien-êtres $W^{A}$ et $W^{D}$ :

$$
W(\lambda)=\int_{q^{I}}^{\bar{q}} W^{D}(q, \lambda) f(q) d q+\int_{\bar{q}}^{\bar{q}} W^{A}(q, \lambda) f(q) d q
$$

L'impact d'une diminution marginale du coût de transport inter-régional est par conséquent le suivant:

$$
\begin{aligned}
\frac{\partial W}{\partial \lambda} & =\underbrace{\left[W^{D}(\tilde{q})-W^{A}(\tilde{q})\right] \frac{d \bar{q}}{d \lambda} f(\tilde{q})}_{\text {Eiffet relcxalisation }} \\
+ & \underbrace{\left[\int_{\tilde{q}}^{\tilde{q}} \frac{\partial W^{A}(q, \lambda)}{\partial \lambda} f(q) d q\right]}_{\text {Eiflet diffusion (CT) }}
\end{aligned}
$$

Cet impact se décompose en deux effets : un effet relocalisation $(R)$ et un effet diffusion $(C T)$.

- L'effet diffusion correspond à l'effet direct de la baisse de $\lambda$ : toutes choses égales par ailleurs, la diminution du coût de transport unitaire facilite la diffusion du bien et se traduit par une baisse des coûts de transport totaux. Cet effet est positif :

$$
C T=-[1-F(\tilde{q})]<0
$$

Une diminution du coût de transport inter-régional se traduit par une diminution des coûts de transport totaux et donc par une augmentation du bien-être.

Il est d'autant plus important que les firmes ont tendance à se concentrer dans la même région. Il est maximum $(C T=-1)$ si la firme entrante se localise toujours dans la région Nord $\left(\tilde{q}=q^{I}\right.$ ou $\left.\lambda<\bar{\lambda}\right)$.

- Une baisse du coût de transport modifie le choix de localisation de la firme entrante et renforce la polarisation géographique. Cet effet relocalisation est négatif du fait de la sur-agglomération des firmes: $W^{A}(\tilde{q})<W^{D}(\tilde{q})$.

Ainsi, lorsque la baisse du coût de transport provoque une augmentation importante de l'agglomération spatiale, l'effet relocalisation d'une forte 
ampleur domine l'effet « diffusion ». En revanche, si le coût de transport devient inférieur à $\bar{\lambda}$, les firmes s'agglomèrent quel que soit la qualité $q$ : l'effet relocalisation disparaît. Dans ce cas, toute baisse du coût de transport a un effet positif sur le bien-être.

L'impact d'une diminution du coût de transport sur les bien-êtres des deux régions peut-être décomposé en deux effets de même nature. Considérons chacune des deux régions.

Dans la région Sud :

$$
\begin{aligned}
& \frac{\partial W S}{\partial \lambda}=\underbrace{\left[W S^{D}(\tilde{q})-W S^{A}(\tilde{q})\right] \frac{d \tilde{q}}{d \lambda} f(\tilde{q})}_{\text {E:fre relocalisation }(\mathbf{R})} \\
& +\underbrace{\left[\int_{\bar{q}}^{\bar{q}} \frac{\partial W S^{A}(q, \lambda)}{\partial \lambda} f(q) d q\right]}_{\text {lilred diffuision (CT) }}
\end{aligned}
$$

- L'effet diffusion correspond, comme précédemment, à l'effet direct de la baisse de $\lambda$ : toutes choses égales par ailleurs, la diminution du coût de transport unitaire facilite la diffusion du bien et se traduit par une baisse des coûts de transport supportés par les consommateurs du Sud.

- Une baisse du coût de transport modifie le choix de localisation de la firme entrante et renforce la polarisation géographique. Cet effet relocalisation est négatif puisque la région Sud pâtit de l'agglomération des firmes : $W S^{A}(\tilde{q})<W S^{D}(\tilde{q})$.

Par conséquent, le Sud risque d'être pénalisé par une baisse du coût de transport si l'effet diffusion est d'une ampleur insuffisante. égal à :

Dans la région Nord, l'effet d'une baisse du coût de transport est

$$
\begin{aligned}
\frac{\partial W N}{\partial \lambda} & =\underbrace{\left[W N^{D}(\tilde{q})-W N^{A}(\tilde{q})\right] \frac{d \tilde{q}}{d \lambda} f(\tilde{q})}_{\text {Eiffer relocalisation (R) }} \\
& +\underbrace{\left[\int_{\tilde{q}}^{\tilde{q}} \frac{\partial W N^{A}(q, \lambda)}{\partial \lambda} f(q) d q\right]}_{\text {Endet diffusion (CT) }}
\end{aligned}
$$

- L'effet diffusion correspond à l'effet direct de la baisse de $\lambda$ : toutes choses égales par ailleurs, la diminution du coût de transport unitaire facilite la diffusion du bien et se traduit par une baisse des coûts de transport qui profite à la firme entrante qui vend le bien aux consommateurs du Sud. Les consommateurs de la région Nord bénéficient de cette augmentation de profit. 
- Une baisse du coût de transport modifie le choix de localisation de la firme entrante et renforce la polarisation géographique. Cet effet relocalisation est positif : l'agglomération des firmes est profitable pour la région Nord : $W N^{A}(\tilde{q})>W N^{D}(\tilde{q})$.

Ainsi, la région Nord profite sans ambiguité d'une baisse du coût de transport.

On peut résumer ces différents résultats dans la proposition suivante.

Proposition 4 Seule une forte baisse du cồt de transport inter-régional assure une augmentation du bien-être total et est sucseptible d'être efficace au sens de Paréto. Une diminution modeste peut au contraire se traduire par une baisse du bien-être total.

Preuve Annexe C

口

W

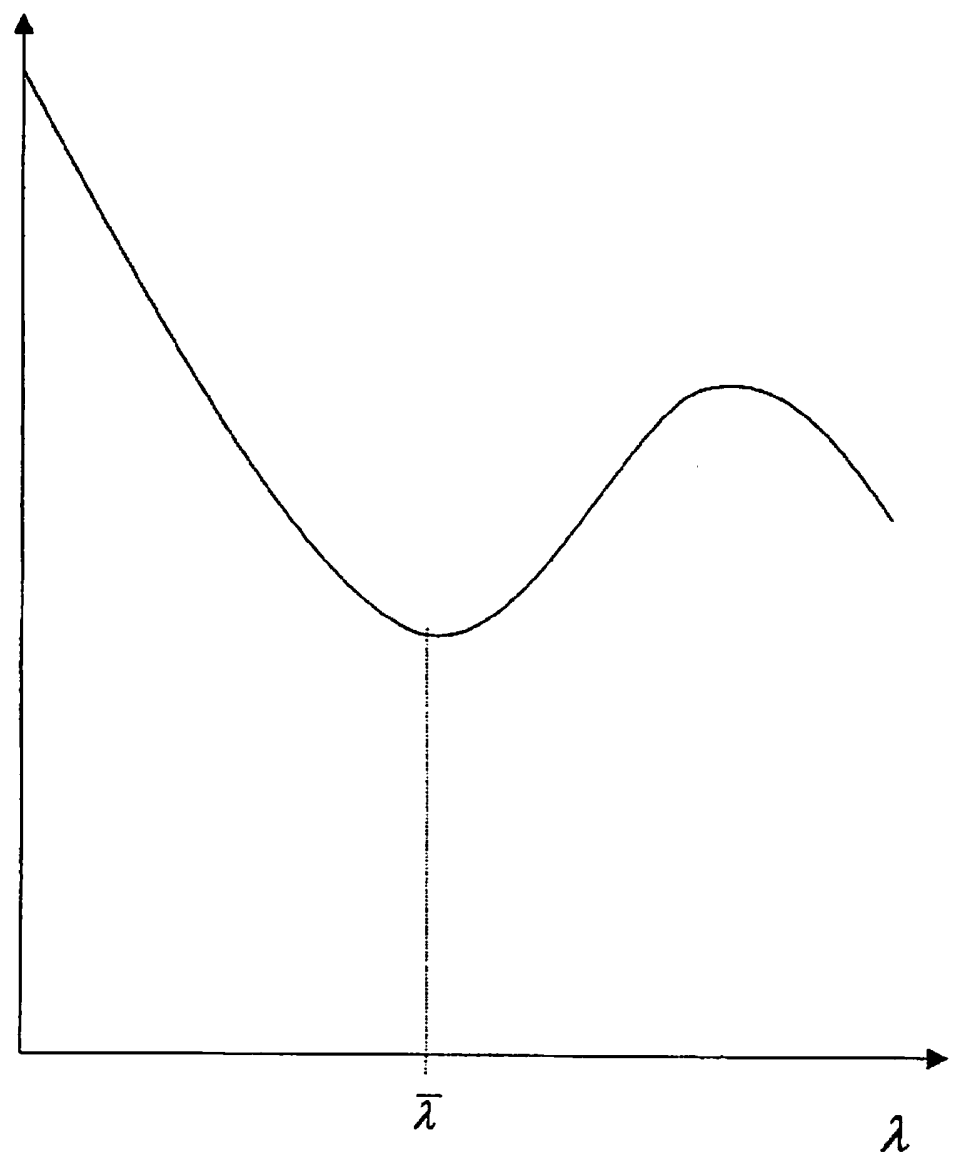

Figure 6 : Bien-être 
La figure 6 représente le bien-être total en fonction du coût de transport inter-régional. On constate que pour certains intervalles du coût de transport, une diminution du coût de transport se traduit par une baisse du bien-être total ${ }^{13}$. En effet, en négligeant l'effet de réputation dû à un manque d'information de la part des consommateurs, l'autorité nationale ne perçoit pas l'effet de seuil de la baisse du coût de transport. Cela comporte deux risques.

Si le coût de transport initial est élevé, une politique de transport d'une ampleur insuffisante pour contre-balancer la polarisation de l'activité peut se révéler néfaste. En effet, en présence d'asymétrie d'information, une faible baisse du coût de transport peut provoquer une forte polarisation de l'activité dans la région Nord. Or si la firme entrante se localise dans la région Nord à la suite de la baisse du coût de transport, développer l'infrastructure de transport pour améliorer la diffusion des biens peut paradoxalement se traduire par une augmentation des coûts de transport totaux. Ainsi d'une part la baisse du coût de transport, en augmentant l'agglomération des firmes, renforce les inégalités entre les deux régions puisque l'agglomération pénalise la région Sud au profit de la région Nord. Mais, d'autre part, les gains engrangés par le Nord ne compensent pas les pertes du Sud. Seul un désenclavement important est alors susceptible d'améliorer le bien-être total de l'économie.

Si l'innovation anticipée est importante, les firmes se localisent toujours dans la région Nord. Une politique publique qui ne tiendrait alors pas compte des asymétries d'information peut au contraire sous-évaluer l'impact toujours positif dans ce cas de figure, d'une baisse du coût de transport. En effet dans ce cas, l'activité étant entièrement localisée dans le Nord, les coûts de transport totaux sont élevés. Une baisse du coût unitaire a alors un impact marginal supérieur à ce qu'il serait si les entreprises étaient dispersées dans l'espace comme c'est effectivement le cas en information parfaite. Parmi les bénéficiaires de l'infrastructure figurent alors paradoxalement les habitants de la région Sud. La baisse des coûts de transport permet aux consommateurs de la région Sud de profiter à moindre coût des biens.

Les politiques de désenclavement peuvent présenter le risque de marginaliser la région Sud. Toutefois une façon de limiter ce risque est d'entreprendre paradoxalement une nette diminution des coûts de transport. Certes, une telle politique ne se traduit pas par une implantation des firmes dans le Sud, mais la meilleure desserte améliore la diffusion de biens vendus par des entreprises qui, de toutes façons, ne se seraient pas localisées dans la région Sud. Par conséquent, une baisse importante du coût de transport peut se traduire par une augmentation du bien-être de la région Sud, qui profite alors du bien à moindre coût. Par conséquent, si le décideur public

13 Ce résultal est certes en partie dú à la discontinuité de l'espace. Notons toutefois que dans un modèle à la Martin et Rogers (1995), l'économie est égalemement composée de deux seules régions et malgré cela, une baisse du coût de transport ne provoque jamais de baisse de bien-être. 
retient un critère de bien-être à la Rawls ou Parétien, seule un fort désenclavement peut se justifier.

\section{Conclusion}

Cet article propose une explication supplémentaire à l'agglomération spatiale des entreprises et plus particulièrement des entreprises innovantes. La proximité spatiale permet aux firmes innovantes de se signaler comme telles. On met ainsi en évidence un équilibre unique dans lequel les firmes les plus innovantes se localisent dans la région où se situent déjà des firmes concurrentes alors que les autres firmes préfèrent éviter toute concurrence au risque de révéler ainsi une innovation médiocre. La concurrence est la source d'un effet de réputation pour la région dont profitent les entreprises. La polarisation de l'activité est par conséquent nettement plus importante que ce qu'elle serait en information parfaite. Par ailleurs une diminution du coût de transport favorise la polarisation spatiale des entreprises. Dans ces circonstances, un faible développement de l'infrastructure de transport se traduit par une baisse du bien-être. On montre qu'il est alors plus efficace d'encourager la construction d'infrastructure de désenclavement conduisant à une forte diminution du coût de transport. Une telle politique ne permet certes pas d'encourager la dispersion de l'activité mais permet au moins un meilleur accès aux innovations.

Trois prolongements de ce modèle peuvent être envisagés. Le premier consiste à étudier l'impact d'une diminution du coût de transport propre à chacune des deux régions. Une deuxième extension viserait à développer ce modèle dans un cadre plus général (à la Ottaviano, Tabuchi et Thisse (2002) par exemple) de façon à endogénéiser, entre autres, le revenu des agents. Le troisième revient à développer un vrai modèle d'innovation dans lequel serait intégrée la décision des firmes d'engager des dépenses de recherche. 


\section{Annexes}

\section{Annexe A}

Démonstration de l'équilibre en prix en information parfaite

Nous traitons ici le cas où la firme $E$ est localisée en $N$. L'autre cas se traite de façon similaire.

On note $\pi_{j}^{i}(i=E, I, j=N, S)$ le profit réalisé par la firme $i$ sur le marché $j$, on obtient les expressions suivantes ${ }^{14}$ :

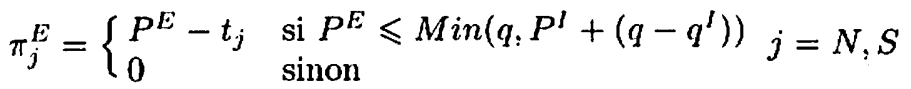

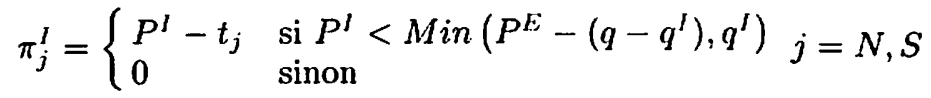

On en déduit les prix d'équilibre présentés page 6 .

\section{Démonstration du lemme 1}

Considérons le cas d'une firme entrante qui supporte des coûts de transport $t^{E}$ pour servir le marché lorsque le coût de transport supporté par la firme concurrente $t^{I}$ est inférieur à $q^{I}$. Les deux entreprises sont alors en concurrence. Supposons que si la qualité de la firme entrante est élément de l'ensemble $Q(e)$, cette dernière se localise en $e$. Un équilibre parfaitement séparateur existe si le prix fixé permet à la firme entrante de signaler sa réelle qualité.

En notant $P(q)$ le prix fixé par la firme de qualité $q$, son profit à l'issue de l'étape 2 est le suivant:

$$
\Pi(P(q))= \begin{cases}P(q)-t^{E} & \text { si } P(q) \leqslant\left(q-q^{l}\right)+P^{I} \\ 0 & \text { sinon }\end{cases}
$$

Or, un équilibre séparateur impose que quelle que soit sa qualité la firme entrante réalise un même profit en étape 2 sous peine de déviation. Un candidat équilibre séparateur est alors nécessairement du type:

$$
P(q)=\left(q-q^{I}\right)+P^{I}+\varepsilon, \quad \varepsilon>0
$$

Premier cas : la firme installée pratique un prix $P^{I}>t^{I}$. On montre qu'aucune croyance hors équilibre ne peut soutenir un tel équilibre si la firme entrante dévie et fixe un prix $P(q)=P^{l}$. En effet dans ce cas le profit

14 On suppose ici que larsqu'un consommateur est indifférent il consomme le bien de meilleure qualité. 
obtenu est égal à $\left(P^{I}-t^{E}\right)$ et ce quelle que soit la qualité anticipée par les consommateurs. Si les deux firmes sont localisées dans la même région, $t^{E}=t^{I}$ et par conséquent $\left(P^{I}-t^{E}\right)>0$.

Deuxième cas : $P^{I}=t^{I}$.

Si la firme entrante est localisée en $S$, sur le marché sud: $t^{E}=0$ et $t^{I}=\lambda$. Aucune croyance hors équilibre ne peut soutenir un tel équilibre si la firme entrante dévie et fixe un prix $P(q)=t^{l}$

Si la firme entrante est localisée en $N$ et si la plus petite qualité élément de $Q(N)$ est différente de $q^{I}$, on montre qu'aucune croyance hors équilibre ne peut soutenir un tel équilibre si la firme entrante dévie et fixe un prix $P(q)=t^{t}+\varepsilon$. En effet quelle que soit la qualité anticipée la firme entrante réalise un profit égal à $\varepsilon>0$. Si la plus petite qualité élément de $Q(N)$ est égale à $q^{I}$, le candidat équilibre séparateur en prix est un équilibre. La firme entrante réalise un profit nul. Toutefois dans ce cas, aucune croyance ne peut dissuader une déviation vers la région Sud où, quelle que soit la qualité anticipée, si $P(q)=t^{I}$, le profit réalisé sera égal à $t^{I}-t^{E}=\lambda$ sur le marché Sud.

Il n'existe donc pas d'équilibre parfaitement séparateur.

Démonstration du lemme 2

Si $q>\lambda+q^{I}$

$$
\begin{gathered}
\frac{\partial \Pi\left(N, P_{2}^{E}, q(.)\right)+\Pi\left(N, P_{3}^{*, E}, q\right)}{\partial q}=2=\frac{\partial \Pi\left(S, P_{2}^{E}, q(.)+\Pi\left(S, P_{3}^{E . *}, q\right)\right.}{\partial q} \\
\quad \operatorname{Si} \lambda<q<\lambda+q^{I}, \\
\frac{\partial \Pi\left(N, P_{2}^{E}, q(.)\right)+\Pi\left(N, P_{3}^{* . E}, q\right)}{\partial q}=2 \geqslant 1=\frac{\partial \Pi\left(S, P_{2}^{E}, q(.)+\Pi\left(S, P_{3}^{E . *}, q\right)\right.}{\partial q} \\
\quad \operatorname{Si~} q<\lambda, \\
\frac{\partial \Pi\left(N, P_{2}^{E}, q(.)\right)+\Pi\left(N, P_{3}^{* . E}, q\right)}{\partial q}=1=\frac{\partial \Pi\left(S, P_{2}^{E}, q(.)+\Pi\left(S, P_{3}^{E . *}, q\right)\right.}{\partial q}
\end{gathered}
$$

\section{Annexe B}

(i) $q^{I}>\lambda$.

Il n'existe pas d'équilibre semi-séparateur si la qualité $q^{I}$ n'a jamais intérêt à dévier de la région $N$ vers la région $S$. C'est le cas si :

$$
\left(q^{m}-q^{l}\right)+\left(q^{m}-q^{l}\right)<2 \lambda
$$


i. e. si :

$$
q^{m} \leqslant \lambda+q^{l}
$$

(ii) $q^{I}>\lambda$.

(a) Considérons d'abord le cas où $q^{m} \geqslant \lambda$

De la même façon, il n'existe pas d'équilibre semi-séparateur si :

$$
\left.\left(q^{m}-q^{I}\right)+q^{m}-\lambda \geqslant 2 q^{I}\right)
$$

i. e. si :

$$
q^{m} \geqslant \frac{3}{2} q^{I}+\frac{\lambda}{2}
$$

Cette condition est compatible avec la condition initiale si :

$$
\lambda \geqslant 3 q^{J}
$$

(b) $q^{m} \leqslant \lambda$

Il existe un seul équilibre mélangeant si

$$
\left(q^{m}-q^{l}\right) \geqslant 2 q^{l}
$$

i. e. si :

$$
q^{m} \geqslant 3 q^{l}
$$

Cette condition est compatible avec la condition initiale si :

$$
\lambda \leqslant 3 q^{l}
$$

En résumé, il existe trois cas de figure :

1. $\lambda \leqslant q^{I}$

Il n'existe pas d'équilibre semi-séparateur si

$$
q^{m} \leqslant \overline{q^{m}}=\lambda+q^{\prime}
$$

2. $q^{I} \leqslant \lambda \leqslant 3 q^{I}$

Il n'existe pas d'équilibre semi-séparateur si

$$
q^{m} \leqslant \overline{q^{m}}=\frac{3}{2} q^{I}+\frac{\lambda}{2}
$$

3. $\lambda \geqslant 3 q^{\prime}$

Il n'existe pas d'équilibre semi-séparateur si

$$
q^{m} \leqslant \overline{q^{m}}=3 q^{I}
$$




\section{Annexe C}

On démontre qu'il peut exister des configurations dans lesquelles une faible baisse de $\lambda$ entraîne une baisse du bien-être $W$.

Supposons que les croyances soient fortement aussi concentrées que l'on veut en $q=\alpha$ avec $f(\alpha)=A$.

Par ailleurs soit $\beta$ le coût de transport qui vérifie $: \tilde{q}(\beta)=\alpha$. En choisissant une diminution marginale du coût de transport $\lambda$ au voisinage de $\beta$, on obtient :

$$
\begin{aligned}
\frac{\partial W}{\partial \lambda} & =\left[W^{D}(\alpha)-W^{A}(\alpha)\right] \frac{d \tilde{q}}{d \lambda} f(\alpha)+\int_{\alpha}^{\bar{q}} \frac{\partial W^{A}(q ; \lambda)}{\partial \lambda} f(q) d q \\
& =A\left[W^{D}(\alpha)-W^{A}(\alpha)\right] \frac{d \tilde{q}}{d \lambda}-(1-F(\alpha))
\end{aligned}
$$

Une telle expression est positive si $A$ est choisi suffisamment grand. Le bien être peut donc augmenter à la suite d'une augmentation du coût de transport inter-régional

\section{Bibliographie}

Aghion, P. et P. Howitt (1997), Endogenous Growth Theory, Cambridge (USA), MIT Press.

Audretsch, D. et M. Feldman (1999), "Innovation in Cities : Science-based diversity, specialization and localized competition", European Economic Review, 43, pp.409-430.

Bester, H. (1998), "Quality uncertainty mitigates product differenciation", Rand Journal of Economics, 29, pp.828-844.

Blundell, G. et V. Reenen (1995), "Dynamic Count Data Models of Technological Innovation", The Economic Journal, 105, pp.333-344.

Boyer, M., J-J. Laffont, P. Mahenc et M. Moreaux (1995), "Blockading Entry", Document de Travail IDEI N95/53.

Dumais, G., G. Ellisson and E. Glaeser (1997), "Geographic Concentration as a Dynamic Process", NBER Working Paper $\mathrm{N}^{\circ} 6270$.

Gehrig, E. (1998), "Cities and the Geography of Financial Centers", CEPR Working Paper N 1894.

Glaeser, E. (1997), "Learning in Cities", NBER Working Paper N 6271. Jacobs, J. (1969), The Economy of Cities, New York, Random House.

Jacobs, J. (1984), La Ville et La Richesse des Nations, Montréal, Boréal. Krugman, P., (1991), "History versus Excpectations", Quaterly Journal of Economics, 106, pp.651-667. 
Lieutaud, M. (1991), La Pouille. Un exemple de programmation industrielle dans le Mezzogiorno italien, École française de Rome.

Mailath, G., M. Okuno-Fujiwara et A. Postlewaite (1993), "Belief B Refinements in Signalling Games", Journal of Economic Theory, 60, pp.241276.

Marshall, A. (1920), Principles of Economics, Londres, MacMillan.

Martin, P. et A. Rogers (1995), Industrial Location and Public Infrastructure, Journal of International Economics, 39, pp.335.

OCDE (1996), Réseaux d'entreprises et développement local, Paris, OCDE.

Ottaviano, G., T. Tabuchi et J. Thisse (2002), "Agglomeration and Trade Revisited", International Economic Review, à paraître.

Pinçon M. et M. Pinçon-Charlot (1992), Quartiers bourgeois, quartiers d'affaires, Paris, Le Seuil.

Porter, M. (1998), "Clusters and The New Economics of Competition", Harvard Business Review, 98, pp.77-90.

Quigley, J. (1998), "Urban Diversity and Economic Growth", Journal of Economic Perspectives, 12, pp.127-138.

Rauch, J. (1993), "Does History Matters When it Matters Little? The Case of City-industry Location", Quaterly Journal of Economics, 108, pp.807-843.

Schulz, N. et K. Stahl, (1996), "Do Cosumers Search for the Highest Price? Oligopoly Equilibrium and Monopolistic Equilibrium Optimum in Differenciated Product market", Rand Journal of Economics, 27, pp.542560.

Umbhauer, G. (1994), "Forward Induction, Consistency and Rationality of $\varepsilon-$ Perturbations", in B. Munier \& F. Machina (eds), Models and experiments in risk and rationality, Kluwer Academic Publishers, pp.413438. 